\title{
Quantifying the Signal Quality of Low-cost Respiratory Effort Sensors for Sleep Apnea Monitoring
}

\author{
Fredrik Løberg \\ University of Oslo
}

\author{
Vera Goebel \\ University of Oslo
}

\author{
Thomas Plagemann \\ University of Oslo
}

\begin{abstract}
Obstructive Sleep Apnea (OSA) is a common, but severely underdiagnosed sleep disorder characterized by recurring periods of shallow or paused breathing during sleep. It is our long-term goal to allow people to perform the first step towards a sleep apnea detection at home by utilizing smartphones, low-cost consumer-grade sensors, and data mining techniques. In this work, we evaluate the signal quality of four respiratory effort sensors (BITalino, FLOW, RespiBAN, and Shimmer), using a RIP sensor from NOX Medical as the gold standard. We design a sixteen-minute signal capture procedure to simulate epochs of disrupted breathing, and capture data from twelve (BITalino and Shimmer) and eleven (RespiBAN and FLOW) subjects during wakefulness. Our signal quality evaluation approach is based on the breath detection accuracy metrics sensitivity and positive predictive value (PPV), along with the breath amplitude accuracy metric weighted absolute percentage error (WAPE). These metrics are closely related to how apneic and hypopneic episodes are scored by medical personnel, making it straightforward to reason about their interpretation. Our results show that false breaths are the primary concern affecting the breath detection accuracy of BITalino, Shimmer, and RespiBAN. Respectively, the sensitivity of BITalino, Shimmer, RespiBAN, and FLOW is $99.61 \%, 98.53 \%, 98.41 \%$, and $98.91 \%$. Their PPV is $96.28 \%, 96.58 \%$, $90.81 \%$, and $98.81 \%$. Finally, their WAPE is $13.82 \%, 16.89 \%, 13.60 \%$, and $8.75 \%$. The supine (back) position is consistently showing the overall best signal quality compared to the side position.
\end{abstract}

\section{CCS CONCEPTS}

\section{- Applied computing $\rightarrow$ Health informatics;}

\section{KEYWORDS}

obstructive sleep apnea, respiratory effort sensors, breath detection accuracy, breath amplitude accuracy

\section{ACM Reference Format:}

Fredrik Løberg, Vera Goebel, and Thomas Plagemann. 2018. Quantifying the Signal Quality of Low-cost Respiratory Effort Sensors for Sleep Apnea Monitoring. In 3rd International Workshop on Multimedia for Personal Health and Health Care (HealthMedia'18), October 22, 2018, Seoul, Republic of Korea. ACM, New York, NY, USA, 9 pages. https://doi.org/10.1145/3264996.3264998

Permission to make digital or hard copies of all or part of this work for personal or classroom use is granted without fee provided that copies are not made or distributed for profit or commercial advantage and that copies bear this notice and the full citation on the first page. Copyrights for components of this work owned by others than ACM must be honored. Abstracting with credit is permitted. To copy otherwise, or republish, to post on servers or to redistribute to lists, requires prior specific permission and/or a fee. Request permissions from permissions@acm.org.

HealthMedia'18, October 22, 2018, Seoul, Republic of Korea

(c) 2018 Association for Computing Machinery.

ACM ISBN 978-1-4503-5982-5/18/10 . .\$15.00

https://doi.org/10.1145/3264996.3264998

\section{INTRODUCTION}

Sleep apnea is a common sleep disorder characterized by recurring periods of shallow or paused breathing during sleep. If a breathing disruption causes the oxygen saturation in the blood to become too low, the brain will force an awakening to resume normal breathing. These awakenings are often very brief, making it unlikely for the sufferer to remember continuously waking up at night. Repeated awakenings inhibit deep sleep, resulting in daytime sleepiness and fatigue. If untreated, sleep apnea can lead to severe health implications for the individual, and, in the worst case, even death if the person is unable to wake up. Sleep apnea is also linked to many serious diseases such as diabetes, hypertension (high blood pressure), heart disease, stroke, depression, and anxiety.

Sleep apnea is a very common, yet severely under-diagnosed disorder. It is estimated that around $25 \%$ of all middle-aged Norwegians are at high risk of having obstructive sleep apnea [6], yet approximately $70-80 \%$ of all cases are expected to be undiagnosed [10]. Without a recollection of the nightly awakenings, and the primary symptom being daytime sleepiness, the disorder may easily remain unnoticed.

The gold standard for diagnosing sleep apnea is polysomnography. Polysomnography is a sleep study which requires the subject to spend the night in a sleep laboratory with many physiological sensors attached to the body. This includes sensors for electroencephalography (EEG), electrocardiography (ECG), electromyography (EMG), electrooculography (EOG), respiratory effort from the chest (thorax) and abdomen, nasal airflow, and oxygen saturation $\left(\mathrm{SpO}_{2}\right)$ [13]. Sleeping in an artificial and unfamiliar environment with so many sensors attached to the body can for many people feel very uncomfortable. Consequently, the threshold for a potential patient to seek a diagnosis is high. In addition, this kind of sleep study is very resource demanding as it requires both expensive equipment, a suited laboratory, and trained medical personnel to monitor and analyze the results manually; making it impossible to prescribe polysomnography for everyone at risk of having sleep apnea. Portable monitoring devices have been developed to enable sleep monitoring at home without the guidance of medical personnel. However, the number of sensors is usually not reduced too much, and the recorded signals still need to be manually evaluated by an expert before an eventual diagnosis can be determined.

Instead of providing alternatives to traditional polysomnography, our objective is to allow people to perform the first step towards a sleep apnea diagnosis at home. The core idea is to drastically reduce the cost and number of required sensors by utilizing smartphones, low-cost consumer-grade sensors (e.g., from BITalino [4] or SweetZpot [12]), and data mining techniques. A potential sleep apnea sufferer should be able to buy a relatively cheap sensor and use that along with a smartphone to test for sleep apnea on their own. The recorded data should be analyzed by data mining techniques to 
detect apnea events automatically, and then potentially recommend that the person should visit a physician. Furthermore, a physician should be able to use the recorded data as a foundation to better decide whether full polysomnography should be performed or not.

For this to be realistic, a few requirements need to be fulfilled. Firstly, the equipment needs to be affordable and easy to use. Secondly, the user should not be bound to any specific equipment but be able to choose the specifics (e.g., what kind of sensor/smartphone, etc.) on their own. Thirdly and most importantly, the produced signals must be of adequate quality. In fact, the quality of the signals has a huge impact on the performance of the data mining classifiers [8].

As an initial step towards this goal, we evaluate in this work the signal quality of four affordable respiratory effort sensors: a piezoelectric effort belt (PZT) from BITalino [4], an impedance plethysmography (IP) sensor from Shimmer [11], a respiratory inductance plethysmography (RIP) sensor (RespiBAN) from biosignalsplux [3], and a strain-gauge sensor (FLOW) from SweetZpot [12]. The gold standard sensor for measuring airflow is a pneumotachograph [2]. In other studies, any quality evaluation of respiratory effort sensors is most often conducted with a pneumotachograph as the gold standard. Unfortunately, one of our limitations is that we do not have access to a pneumotachograph, and, therefore, have to measure the signal quality using other means. We use a clinically certified RIP sensor from NOX Medical as the gold standard [9]. Respiratory effort sensors are often belts strapped around the thorax and abdomen measuring the movement associated with breathing. Hence, they are used in the same way as commonplace pulse belts used during exercise, which many people already are quite familiar with. By this, we address the following research questions:

(1) Which metrics are appropriate to measure the signal quality of respiratory effort sensors?

(2) In which setting should we capture the signal data?

(3) How can we measure the signal quality with our limited set of resources?

(4) How good are the BITalino, Shimmer, RespiBAN and FLOW sensors?

To approach these questions, we design a generic sixteen-minute signal capture procedure to simulate epochs of disrupted breathing during wakefulness. With data from twelve (BITalino and Shimmer) and eleven (RespiBAN and FLOW) subjects, we evaluate the signal quality of these sensors by utilizing metrics which are closely related to how apneic and hypopneic episodes are scored by medical personnel; making it trivial to reason about their interpretation. Namely, the breath detection accuracy metrics sensitivity and positive predictive value (PPV), alongside the breath amplitude accuracy metric weighted absolute percentage error (WAPE). Our results show that false breaths are the primary concern affecting the breath detection accuracy of BITalino, Shimmer, and RespiBAN. Respectively, the sensitivity of BITalino, Shimmer, RespiBAN, and FLOW is $99.61 \%, 98.53 \%, 98.41 \%$, and $98.91 \%$. Their PPV is $96.28 \%, 96.58 \%$, $90.81 \%$, and $98.81 \%$. Finally, their WAPE is $13.82 \%, 16.89 \%, 13.60 \%$, and $8.75 \%$. The supine (back) position is consistently showing the overall best signal quality compared to the side position. To the best of our knowledge, there does not exist any comparative study of this kind.

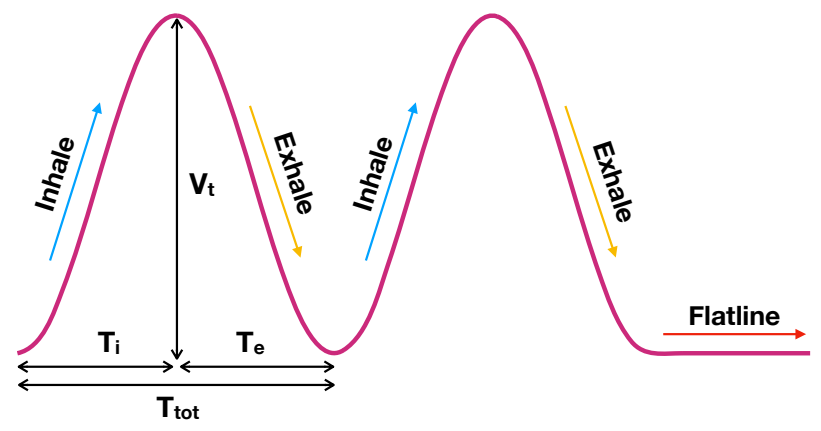

Figure 1: Respiratory effort volume signal

The remainder of this paper is structured as follows: Section 2 describes the used method and Section 3 presents our evaluation results. Section 4 summarizes conclusions and future work.

\section{METHOD}

In this section, we start by describing respiratory effort signals in general. This includes what the signals look like and commonly used physiological features present in the signals. Next, we present the breath detection accuracy metrics and breath amplitude accuracy metric we use during the signal quality evaluation. At last, we present our signal capture procedure which all the subjects undertake.

\subsection{Respiratory Effort Signals}

Before we explain the signal quality metrics, it is important to fully understand the signals themselves. The respiratory effort sensors we evaluate, all measure the abdominal/thoracic expansion and contraction associated with breathing (see Figure 1). The $y$-value increases with the distraction of the belt, or expansion of the thorax and abdomen as the person inhales, and decreases as the belt contracts. The $x$-axis represents time. When the belt distraction is constant over a time period, like when the person stops breathing, the signal flatlines. This kind of signal is known as a volume signal, in other words, the $y$-axis reflects the current stretch of the belt at any given time. Another kind is an airflow signal, which is what both the BITalino and RespiBAN sensors produce. An airflow signal reflects the current flow of air per time unit passing through the airways. This means that as soon as airflow subsides, the signal immediately returns to zero. Visually, both types of signals look very similar, and during normal uninterrupted breathing, they are almost indistinguishable.

From Figure 1, we see that each peak corresponds to individual breaths. A very commonly used global feature of this kind of signal is the number of breaths per minute (BPM), which is derived by counting the number of peaks per minute. Each breath can also be deconstructed into multiple internal features, such as tidal volume $\left(V_{t}\right)$, inspiratory time $\left(T_{i}\right)$, expiratory time $\left(T_{e}\right)$, and total breath duration $\left(T_{t o t}\right)$.

One of the challenges with respiratory effort sensors is that the units of the $y$-axis are relative. The values of the $y$-axis depend very much on how tight the sensor is fitted around the person and also vary greatly from sensor to sensor. As such, the interpretation of 
the $y$-values is not straightforward. Absolute units can be derived by performing a calibration procedure, but studies have shown that when the subjects are allowed to change sleeping position in bed, the calibration is easily invalidated [5], [14]. As such, calibration is rarely done, even in clinical settings [1]. On a side note, unless the sensor is calibrated, the amplitude of a breath is often referred to as breath amplitude rather than tidal volume.

To measure the signal quality of a given sensor, features such as the number of breaths per minute, the breaths themselves, or any of these local features are commonly compared to the same features of a gold standard signal using a wide range of different statistical metrics. However, because the $y$-axis is relative and varies between sensors, the values of the breath amplitudes from two different sensors cannot be compared directly. Either, a metric which supports variables with different units must be used, or the signals must be transformed to the same unit of measurement beforehand. One way this can be done, and also the way we do it, is to use linear regression to derive the linear relationship between the breath amplitude values before the metric is calculated.

Another challenge with respiratory effort signals is that they are per definition not reproducible. It is practically impossible for a person to perform precisely the same movements twice. Thus, the reproducibility relies on the number of recordings and subjects.

2.1.1 Preprocessing. We apply minimal preprocessing in this work. Firstly, we resample the BITalino, Shimmer, and RespiBAN signals to $20 \mathrm{~Hz}$, and the FLOW signals to $10 \mathrm{~Hz} .20 \mathrm{~Hz}$ is the (fixed) sampling rate of the NOX sensor, whereas $10 \mathrm{~Hz}$ is the (fixed) sampling rate of the FLOW sensor. In other words, we downsample the BITalino, Shimmer, and RespiBAN to match the NOX, and the NOX to match the FLOW sensor. Secondly, to synchronize the target sensor signals with the gold standard signal, we use crosscorrelation. See Equation 1, where $\hat{y}$ is the first signal, $y$ the other signal, and $d^{*}$ the displacement index of the synchronization point. The use of cross-correlation for synchronization is meant only as a means to speed up the process, as we validate the synchronization manually regardless. Additionally, the metrics we employ do not impose any strict precision requirements for the synchronization. As long as the peak of a breath is between the start and end of the corresponding breath in the gold standard signal, then the synchronization precision is adequate.

$$
d^{*}=\underset{d \in \mathbb{Z}}{\arg \max }\left(\sum_{i=-\infty}^{+\infty} \hat{y}[i] y[i+d]\right)
$$

Thirdly, we standardize the already relative signals for visual comparability. See Equation 2, where $X$ are the samples of the signal, $\mu$ is the signal's mean value, and $\sigma$ is the signal's standard deviation.

$$
X_{n e w}=\frac{X-\mu}{\sigma}
$$

Finally, we also generate and measure the signal quality of a composite logical signal from the BITalino sensor, namely the sumsignal. The respiratory process can be described as a system with two degrees of freedom (2-DOF) of motion [7]. The sum of the movement from both the thorax and abdomen, after calibration, reflects a semiquantitative estimate of tidal volume. The calculation of RIP sum is shown in Equation 3, where $a$ and $b$ are constants determined as the result of a calibration procedure. One should, however, note that even in clinical settings, calibration of the belts is rarely performed, and hence the uncalibrated version of RIP sum $_{\text {. }}$ is more widely used [1]. Consequently, the constants $a$ and $b$ are usually both set to 0.5 so that the magnitude of the RIP sum signal is comparable to the magnitude of the raw thoracic and abdominal signals. On a side note, RIP $_{\text {sum }}$ is only used as an example, and the same description analogously applies to the PZT counterpart $\mathrm{PZT}_{\text {sum }}$ (and other counterparts). In this work, we set both the $a$ and $b$ constants to 1 as the signals are standardized anyway.

$$
R I P_{\text {sum }}=a \cdot R I P_{\text {thorax }}+b \cdot R I P_{\text {abdomen }}
$$

\subsection{Metrics}

It is important to choose signal quality metrics which measure the factors that are important for sleep apnea monitoring. Anything else is useless for our goal. For a sensor to perform well at detecting sleep apnea, it needs to be able to detect two types of breathing disruptions, apneas and hypopneas.

An apnea is defined as a complete breathing stop lasting a minimum of ten seconds. In other words, an apnea is present in the signal whenever breaths are absent for ten seconds or longer. This means that if a sensor is able to accurately detect both the presence and absence of breaths in general, then it is also able to accurately detect apneas. As a result, we need a metric to represent the breath detection accuracy.

A hypopnea is defined as a minimum of $30 \%$ reduction in airflow, lasting a minimum of ten seconds. In other words, a hypopnea is present whenever the amplitudes of the breaths drop by a minimum of $30 \%$ over ten seconds or longer. This means that if a sensor can accurately detect breaths, and those breaths have an accurate amplitude, then the sensor is also able to accurately detect hypopneas.

Summarized, we need metrics to represent both breath detection accuracy and breath amplitude accuracy.

2.2.1 Breath Detection Accuracy. For the breath detection accuracy, we employ two metrics: sensitivity (also called recall) and positive predictive value (also called precision). Sensitivity measures the proportion of real breaths detected by a sensor, whereas positive predictive value measures the proportion of detected breaths that are real. The general formulas for sensitivity and positive predictive value are shown in Equation 4 and Equation 5, respectively. $T P, F N$, and FP are the number of true positive, false negative, and false positive breaths, respectively. Since it does not make sense to define a true negative breath, the commonly used specificity metric is precluded from this measurement.

$$
\begin{gathered}
\text { sensitivity }=\frac{T P}{T P+F N} \\
\text { positive predictive value }=\frac{T P}{T P+F P}
\end{gathered}
$$

Thereby, a respiratory effort sensor may detect both true and false breaths, and it may also miss breaths. To calculate these two metrics, we regard all the breaths detected by the gold standard sensor as real breaths. Next, any breath in the target sensor signal whose peak is between the start and end of a breath in the gold 
standard signal is matched and thereafter regarded as a true breath Conversely, any unmatched breath is regarded as false. Sensitivity is calculated with the number of breaths in the gold standard signal as the denominator (i.e., $F P+F N$ ) and the number of matched breaths from the target sensor signal as the numerator (i.e., TP). Positive predictive value is calculated with the total number of breaths in the target sensor signal as the denominator (i.e., $T P+F P$ ) and the number of matched breaths as the numerator (i.e., $T P$ ).

2.2.2 Breath Amplitude Accuracy. For hypopneas, the minimum $30 \%$ reduction in airflow threshold is calculated relative to the baseline amplitude of the normal breaths. Both this baseline and the relative airflow reduction is most often subjectively identified visually. For our measurements, we regard the mean amplitude of the breaths to be this baseline. For the breath amplitude accuracy, we utilize the weighted absolute percentage error (WAPE) metric (also known as the $M A D /$ mean ratio). The formula is shown in Equation 6, where $y$ are the breath amplitudes of the gold standard, $\bar{y}$ is the mean gold standard breath amplitude, $\hat{y}$ are the breath amplitudes of the target signal, and $n$ is the number of breaths.

As previously mentioned, the unit of measurement between different sensors varies. Therefore, we use Theil-Sen linear regression of the breath amplitudes beforehand to transform them to the same unit of measurement.

$$
\begin{array}{r}
n=|y| \\
W A P E=\frac{1}{n} \sum_{i=0}^{n} \frac{\left|\hat{y}_{i}-y_{i}\right|}{\bar{y}} \times 100 \%
\end{array}
$$

Several other studies evaluate the signal quality of respiratory sensors based on either the signal as a whole or the accuracy of each breath in isolation. The result is that many aspects of the signals that are irrelevant in the context of sleep apnea monitoring are still included in the signal quality evaluation. We instead employ metrics which are closely related to how medical personnel scores apneic and hypopneic episodes. Apneic episodes are ultimately scored based on the absence of breaths. It is, therefore, only false and missing breaths that affect a sensor's ability to detect apneic events.

\subsection{Signal Capture Procedure}

Signal capture sessions performed overnight while the subjects are asleep, including both healthy as well as sleep apnea patients, are with no doubt the most representative sessions for sleep apnea monitoring. The reason is that there are certain events and characteristics of these kinds of sessions that are very likely to influence the results of the signal quality evaluation. For example, given a sensor which simply cannot flatline. In other words, when a subject stops breathing, the sensor starts to act weirdly and produces events that can be regarded as breaths. If breathing stops are not included in the signal capture procedure, such an issue will not be discovered. These longer overnight sessions, however, require significantly more work, both with respect to execution as well as to subject recruitment, compared to shorter sessions that can be performed in a laboratory during wakefulness. A decent quantity of signal captures is also essential to be able to generalize about the results. As such, shorter sessions that can be performed in a laboratory during wakefulness are preferred, as long as they are sufficiently representative. In this work, we, therefore, set out to simulate periods of disrupted breathing through shorter wakeful sessions.

The setting of the signal capture procedure is, as a result, set in bed with two different body positions while the subject is simulating periods of disrupted breathing. During a normal night's sleep, we have four core sleeping positions. One can lie on the stomach, back (supine), or either side. Because the equipment is placed on the chest, which makes it very uncomfortable to lie on the stomach, we are left with the supine and side body positions for these experiments.

A sleep apnea patient is likely to experience both apneic and hypopneic events, as well as periods of deep breathing as the person may be gasping for air. All these multiple times throughout a night. Both the apneic and hypopneic events are at least ten seconds in duration, as of their definitions, which means that our simulated versions also need to be at least ten seconds.

We simulate apneic events by not breathing, by holding the breath. This should result in a flatline in the signal. To simulate hypopneic events, the person needs to inhale at least $30 \%$ less air on each breath over ten seconds or more. This kind of breathing is not easy to do, so there will be inevitable differences between the subjects' performance.

Considering the duration of the signal captures, the shorter, the better, because it can easily become very boring for the subjects if it is too tedious. Nevertheless, we still need to be able to capture the general case for the captures and measurements to be useful. We know that the signal captures should at least include the following: two body positions, where each position includes a period of deep, shallow, and no breathing. Also, these periods of disrupted breathing need to be at least ten seconds in duration.

To further substantiate the choice of duration, we conduct several preliminary tests to see if there are any apparent changes to the signal quality over time. What we found during the preliminary testing is that the first two minutes of the signal is often very noisy. This is the case even for the NOX sensor. The cause, we believe, is a combination of movement from the subject and initial internal calibration of the sensors. As a result, we ended up with a sixteen-minute procedure. With the first two minutes removed, seven minutes are left for each of the two body positions and their respective periods of disrupted and normal breathing.

The final signal capture procedure which all the subjects performed is thereby as follows:

- Minute 1-9: Subject lies in the supine position.

- Minute 3: Subject holds their breath for 17 seconds.

- Minute 4: Subject holds their breath for 17 seconds.

- Minute 5: Subject breathes shallowly for 17 seconds.

- Minute 6: Subject breathes deeply for 17 seconds.

- Minute 10-16: Subject lies in the side position.

- Minute 12: Subject holds their breath for 17 seconds.

- Minute 13: Subject holds their breath for 17 seconds.

- Minute 14: Subject breathes shallowly for 17 seconds.

- Minute 15: Subject breathes deeply for 17 seconds.

During the first nine minutes, the subject lies in the supine body position and performs four actions spread throughout the duration (except in the noisy first two minutes). After these nine minutes, the 


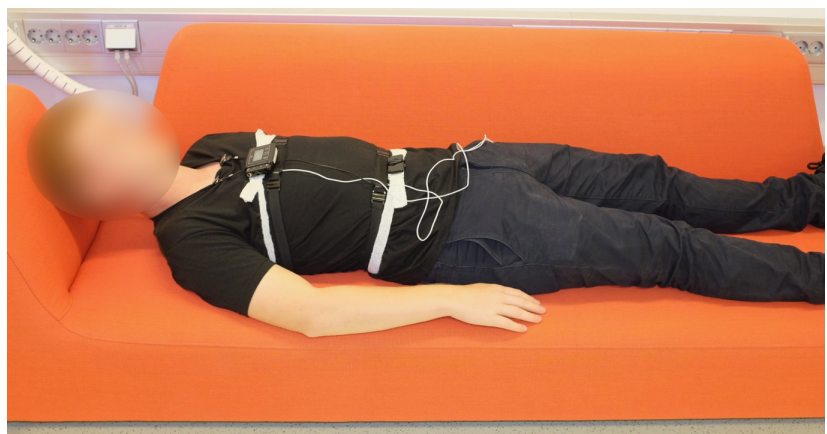

Figure 2: BITalino (black) and NOX (white) belts on a subject

subject turns to the side position, and performs the same actions again. In-between the periods of disrupted breathing, the subject lies completely still while breathing normally.

This signal capture procedure is generic and not specifically designed for the respiratory effort sensors we are evaluating. It is designed for any sensor that directly monitors the respiratory process, such as an oronasal thermal sensor, nasal pressure transducer, and most types of respiratory effort sensors. The procedure may be used with sensors that indirectly monitor the respiratory process as well, such as a pulse oximeter or ECG, but the duration of the periods of disrupted breathing may need to be adjusted. For example, a period of 10-20 seconds may not be long enough to affect the $\mathrm{SpO}_{2}$ levels as much as necessary.

\section{EVALUATION}

In this section, we present the evaluation of the signal quality of the four sensors in question. We start by showing some example signals from the subjects, before we continue by presenting the breath detection accuracy and breath amplitude accuracy. Next, we describe our experience of integrating the airflow signals, and finally, we present several takeaway points.

Preliminary testing shows that all sensors perform reasonable consistent between different recordings. The basic experimental set-up is to use the NOX and the sensor under evaluation in one recording (see Figure 2). To increase the comparability of the sensor performances, we recorded some of the signals in parallel: BITalino \& Shimmer, and FLOW \& RespiBAN. In other words, the subjects wore the BITalino, Shimmer, and NOX sensors during one session, and then the RespiBAN, FLOW, and NOX sensors during another session. As a result, all signals are evaluated separately but share the gold standard signal with one other sensor. Nonetheless, a thorough investigation of re-test reliability is subject to future work. As mentioned above, due to the nature of respiratory effort signals, the reproducibility relies solely on the number of recordings and subjects.

\subsection{Signal Examples}

Figure 3 presents examples of what the signals under evaluation look like. An example from the gold standard sensor NOX is also included for reference. Please note that all signals are standardized, which means that the amplitudes are relative and, therefore, vary between the signal captures.

\begin{tabular}{|l|r|c|c|}
\hline & Sensitivity & PPV & WAPE \\
\hline $\begin{array}{l}\text { BITalino } \\
\text { (abdomen, supine) }\end{array}$ & $\mathbf{9 9 . 6 1 \%}$ & $96.28 \%$ & $13.82 \%$ \\
\hline $\begin{array}{l}\text { BITalino } \\
\text { (abdomen, side) }\end{array}$ & $99.16 \%$ & $93.83 \%$ & $16.51 \%$ \\
\hline $\begin{array}{l}\text { BITalino } \\
\text { (thorax, supine) }\end{array}$ & $97.47 \%$ & $94.69 \%$ & $20.60 \%$ \\
\hline $\begin{array}{l}\text { BITalino } \\
\text { (thorax, side) }\end{array}$ & $97.81 \%$ & $92.24 \%$ & $22.36 \%$ \\
\hline $\begin{array}{l}\text { BITalino } \\
\text { (sum, supine) }\end{array}$ & $99.48 \%$ & $96.44 \%$ & $14.28 \%$ \\
\hline $\begin{array}{l}\text { BITalino } \\
\text { (sum, side) }\end{array}$ & $98.53 \%$ & $96.58 \%$ & $16.89 \%$ \\
\hline $\begin{array}{l}\text { Shimmer } \\
\text { (thorax, supine) }\end{array}$ & $97.30 \%$ & $97.55 \%$ & $21.37 \%$ \\
\hline $\begin{array}{l}\text { Shimmer } \\
\text { (thorax, side) }\end{array}$ & $98.41 \%$ & $90.81 \%$ & $13.60 \%$ \\
\hline $\begin{array}{l}\text { RespiBAN } \\
\text { (abdomen, supine) }\end{array}$ & $98.98 \%$ & $86.64 \%$ & $14.65 \%$ \\
\hline $\begin{array}{l}\text { RespiBAN } \\
\text { (abdomen, side) }\end{array}$ & $98.81 \%$ & $\mathbf{8 . 7 5 \%}$ \\
\hline $\begin{array}{l}\text { FLOW } \\
\text { (abdomen, supine) }\end{array}$ & $\mathbf{9 9 . 1 6 \%}$ & $9.61 \%$ \\
\hline $\begin{array}{l}\text { FLOW } \\
\text { (abdomen, side) }\end{array}$ & $98.51 \%$ \\
\hline
\end{tabular}

Table 1: Overview of the mean metric scores of the signals

As presented above, the signal capture procedure consists of four actions per body position, two periods of no breathing, followed by a period of shallow breathing, followed by a period of deep breathing. All of these periods last for seventeen seconds and are separated by slightly longer periods of normal breathing. Exactly when these periods occur during the signal captures are annotated in the examples shown in Figure 3. A common feature visible in these examples is a deeper breath at the end of the periods of disrupted breathing, as the subjects are gasping for air. Another point one may notice is that the baseline of both BITalino and RespiBAN is centered in the middle of the signal, indicating that both of these are airflow signals. Conversely, Shimmer, FLOW, and NOX all produce a volume signal.

\subsection{Breath Detection Accuracy}

We recruited and gathered data from twelve subjects for BITalino and Shimmer, and eleven subjects for RespiBAN and FLOW. Table 1 shows the mean score of each metric for all signals, with the best performing signal emphasized.

The total number of real breaths for each of these signal captures is in the range of $80-130$, which means that each missing breath results in a sensitivity loss of about $1.25-0.76 \%$. Please note that sensitivity alone does not directly imply that the signal is of good 
(a) BITalino

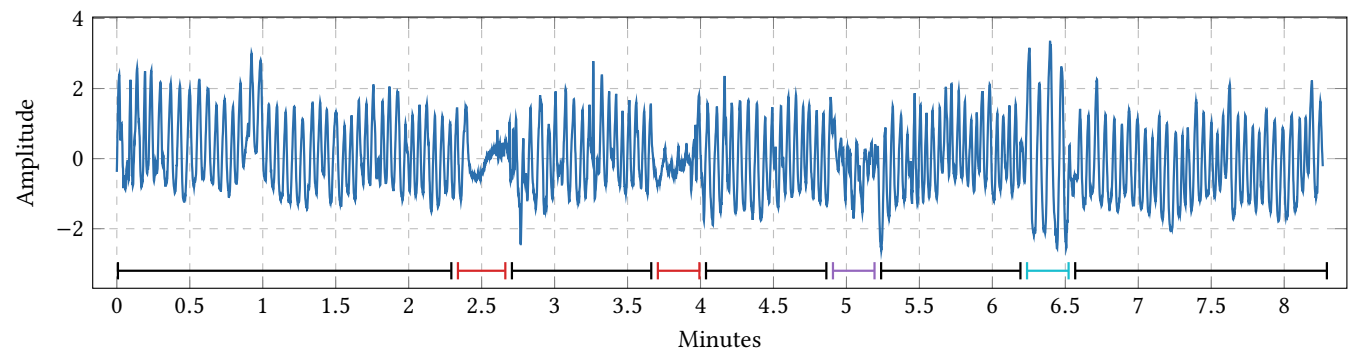

(b) Shimmer

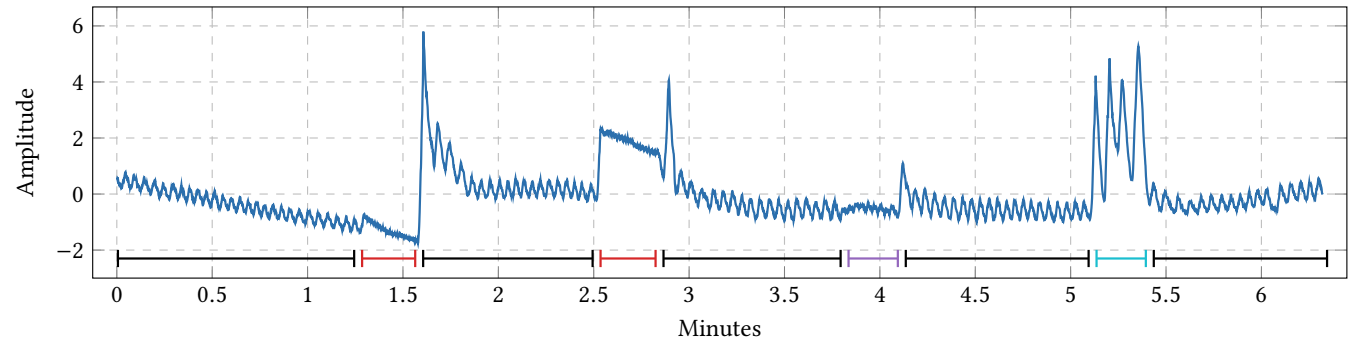

(c) RespiBAN

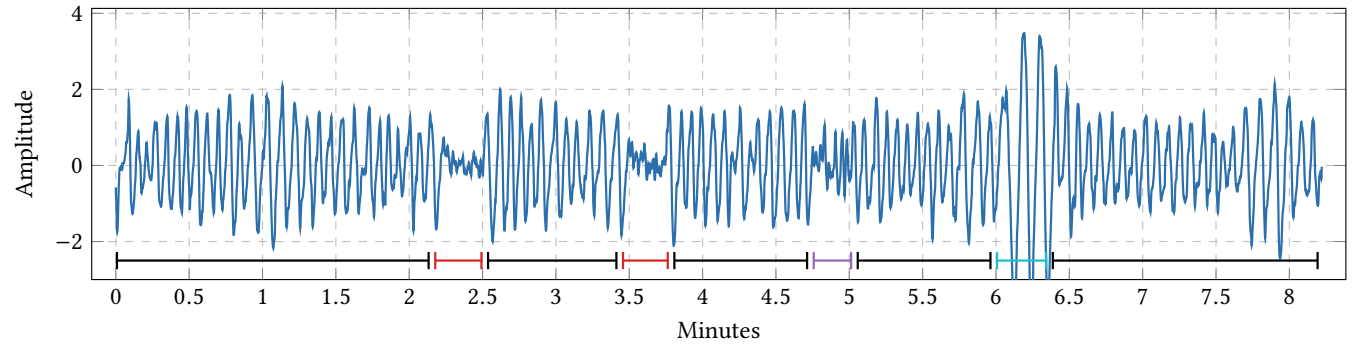

(d) FLOW

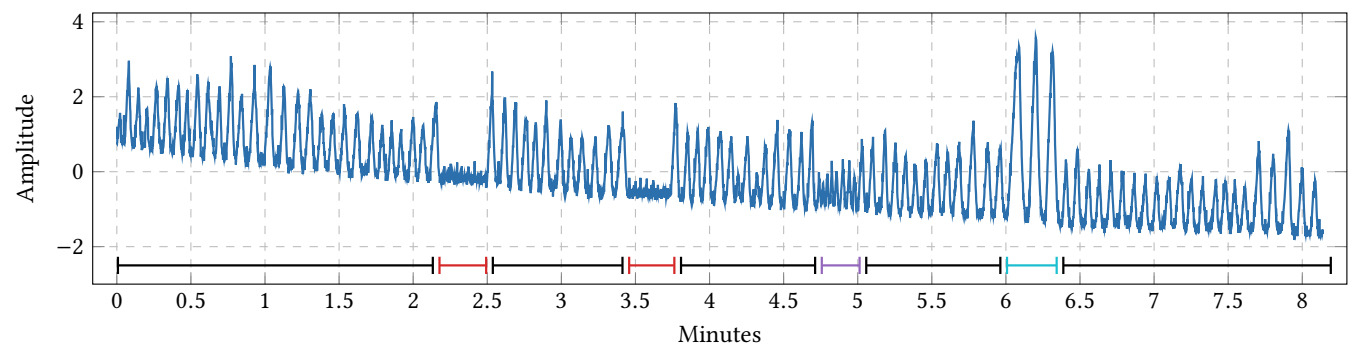

(e) NOX

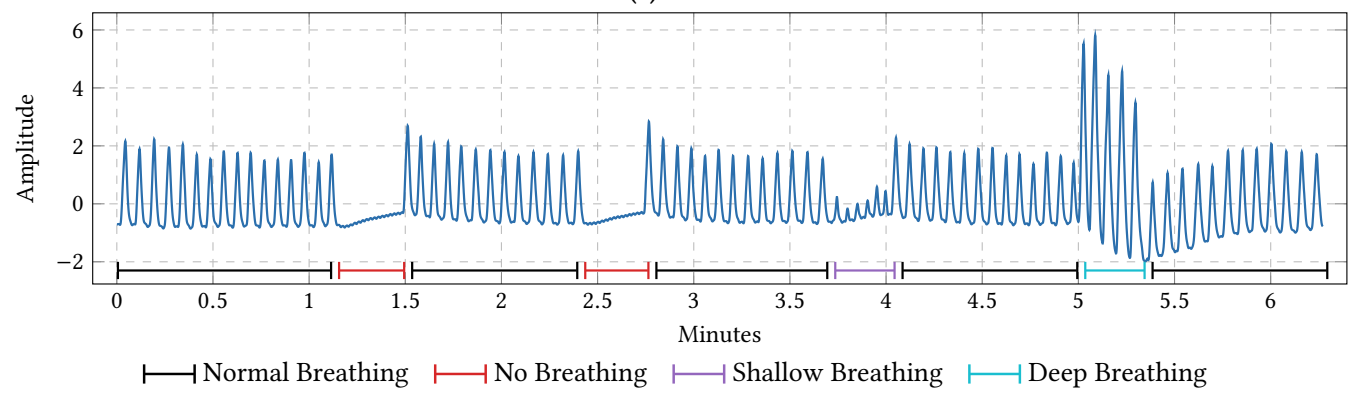

Figure 3: Example of signals from the sensors 
quality. A large number of false breaths increases the odds of false breaths being identified as true, and may, thus, also result in a higher sensitivity. Whereas a large number of false breaths may increase sensitivity, it may also make it significantly more challenging to detect epochs of disrupted breathing.

As seen in Table 1, the BITalino sensor, which is also the least expensive one, has a clearly better sensitivity than all the other sensors. They are not far behind, but none of them even reach $99 \%$ or more, while all abdominal signals from BITalino do. The missing real breaths of the sensors are often caused by the shallow breaths being buried in noise. This is sometimes caused by the breath amplitudes being too low in general (low signal-to-noise ratio), which buries even the normal breaths in noise. One may notice that the sensitivity is better in the supine position for most of the signals. All in all, the sensitivity is rather good for all sensors.

Next is the positive predictive value (PPV). Unlike sensitivity, the PPV does not decrease linearly as false breaths are added to a signal because the number of false breaths is a part of the denominator rather than the numerator. The lower the PPV, the more false breaths are present.

From the PPV, we can see that the good sensitivity of BITalino is actually caused by it being over-sensitive, resulting in many false breaths. These false breaths are mostly present during the periods of disrupted breathing, while also sometimes during normal breathing. For Shimmer and FLOW, the PPV and sensitivity are somewhat balanced. One may notice the PPV of RespiBAN, which is the poorest score of these sensors, despite RespiBAN being the most expensive sensor. The problem with RespiBAN is not during breathing, but that it simply cannot flatline, resulting in many false breaths during the simulated apneic events.

\subsection{Breath Amplitude Accuracy}

The weighted absolute percentage error (WAPE) metric calculates the error (or distance), which means that the lower the metric score, the higher the accuracy, I.e., lower is better. One must note that the result of entirely random data for this metric is $50 \%$, which means that anything close to or worse than this may correspond to an inferior performance depending on the underlying distribution.

For the BITalino sensor, one may notice that the thoracic signal is significantly worse than the abdominal. It is, in fact, more than twice as bad for seven out of twelve subjects in the supine position. Overall, it is worse than the abdominal signal for nine out of twelve and seven out of ten subjects for the supine and side positions, respectively. In the few other cases, it is either equal to or only slightly better than the abdominal signal.

For this metric, there is a clear difference between the supine and side body positions. The supine position is significantly better for most of the signals for all the sensors. The FLOW sensor has the most accurate amplitudes overall, with the supine signal from RespiBAN and BITalino not too far behind.

The sum of the abdominal and thoracic signals amplifies features that are common to both signals (i.e., breaths) and minimizes the features that are unique to one of the signals (i.e., noise). The expected outcome is that the sum-signal should perform better than the abdominal and thoracic signals alone. However, whereas the sum-signal is overall very good, it is also rarely better than both the raw abdominal and thoracic signals but lies more often somewhere between the two.

Figure 4 shows examples of the breath amplitude relationship of the sensors. This figure shows only an example from each sensor, and the relationship does vary between captures. The relationships of the breath amplitudes of the target sensors to the NOX are often monotonic, rather than linear, and the slope of the relationship is not consistent across different subjects or signals.

\subsection{Integrating Airflow Signals}

Both the BITalino and RespiBAN sensors capture the respiratory process as airflow (despite the technology of RespiBAN, RIP, implying that it should capture volume). As of this fact, one would expect that integrating these signals should result in a better WAPE score compared to the raw signals. This is, however, not the case. We cumulatively integrate these signals using the common trapezoidal rule, but the integrated versions score almost always significantly worse than their raw counterparts. We suspect that the underlying cause includes noise and measurement errors regarding breath amplitude and duration, which are amplified by the integration.

Integration acts as a low-pass filter, which effectively attenuates high-frequency noise, smoothing the signal. Whenever the signal-to-noise ratio is better, integration is an effective method to minimize the number of false breaths. However, as a good signal-tonoise ratio is generally not the norm, integration does more harm (to sensitivity) than good (to PPV).

\subsection{Main Results}

For both BITalino and Shimmer, the supine position shows the superior results. For the BITalino sensor, it is the raw abdominal signal from the supine position that shows the best results overall. This signal shows on average a sensitivity, PPV, and WAPE score of $99.61 \%, 96.28 \%, 13.82 \%$, respectively. Of all signal combinations, this signal achieves the best sensitivity metric score. Whereas the raw signals from BITalino achieve better scores overall compared to their integrated counterparts, the integrated versions achieve the best PPV scores. The reason is that integration acts as a low-pass filter, which effectively attenuates the false breaths from the signal. The primary signal quality concern for BITalino is the presence of false breaths. A large number of false breaths is expected to increase the rate of false negative apneic and hypopneic events. Of 48 raw signals (two from each subject from each body position), three are corrupt. Two thoracic signals and one abdominal signal, both from the side position.

As mentioned, the best performing signal from Shimmer is from the supine position as well. This signal achieves on average a sensitivity, PPV, and WAPE score of $98.53 \%, 96.58 \%, 16.89 \%$, respectively. The signal quality of the Shimmer sensor is less stable between subjects compared to the BITalino sensor. In other words, the signal quality of Shimmer is often either very good or somewhat poor, but seldom in between. While the BITalino sensor struggles mainly with false breaths, the Shimmer sensor is also somewhat concerned with missing breaths. Out of 24 signals from Shimmer, four are corrupt. Three of those are from the side position of subjects with a BMI above 30, while one is from the supine position of a subject with an average BMI. There is possibly a trend related to signal 
(a) BITalino

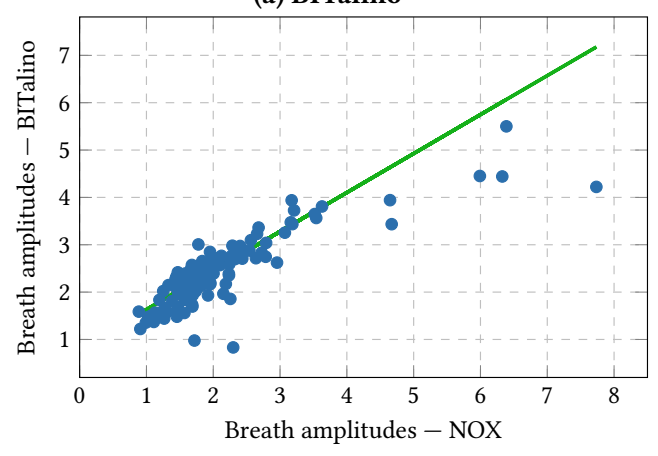

(c) RespiBAN

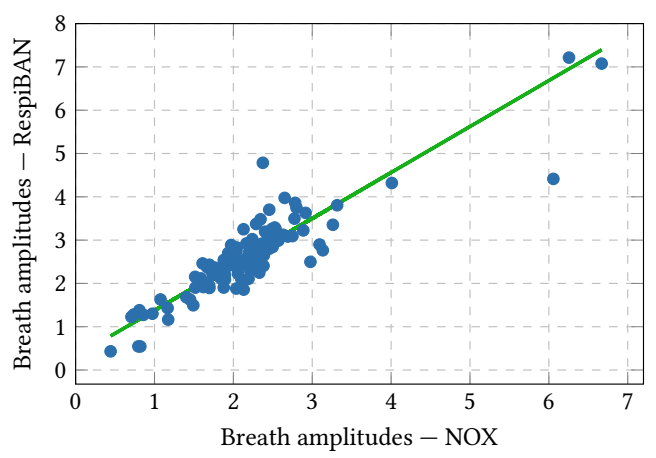

(b) Shimmer

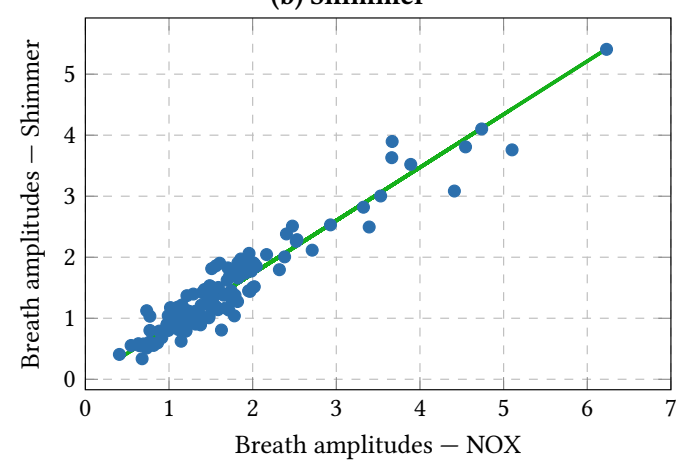

(d) FLOW

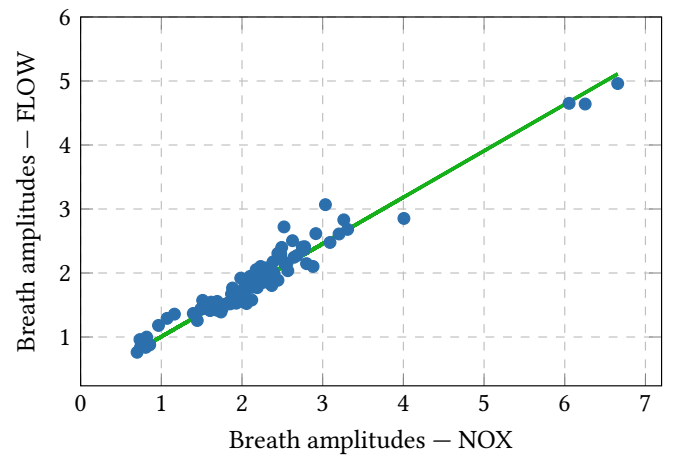

Regression line

Figure 4: Breath amplitude relationship between the target sensors and NOX

quality and BMI present in the data. The signal quality is worse on both ends of the BMI scale, and best close to a BMI of 25 (i.e., average).

Compared to BITalino and Shimmer, the signal quality from both the RespiBAN and FLOW is remarkably stable across different signal captures, subjects, and body positions. There are, in other words, far fewer outliers for these sensors. The RespiBAN sensor is severely struggling with false breaths during breathing stops because the signal just cannot flatline. The FLOW sensor is not struggling with anything in particular related to the signal quality metrics, but the signal is, nonetheless, very noisy. The supine signal from RespiBAN achieves on average a sensitivity, PPV, and WAPE metric score of $98.41 \%, 90.81 \%$, and $13.60 \%$, respectively. Likewise, the supine signal from FLOW achieves on average a sensitivity, PPV, and WAPE metric score of $98.91 \%, 98.81 \%$, and $8.75 \%$, respectively.

\section{CONCLUSIONS}

In this work, we evaluate the signal quality of four respiratory effort sensors for sleep apnea monitoring. Namely, a piezoelectric effort belt (PZT) from BITalino, an impedance plethysmography (IP) sensor from Shimmer, a respiratory inductance plethysmography (RIP) sensor (RespiBAN) from biosignalsplux, and a strain-gauge sensor (FLOW) from SweetZpot. We use a RIP sensor from NOX Medical as the gold standard. To evaluate the signal quality of these sensors, we design a sixteen-minute signal capture procedure and capture data from twelve (BITalino and Shimmer) and eleven (RespiBAN and FLOW) subjects. Our signal quality evaluation approach is based on the breath detection accuracy metrics sensitivity, and positive predictive value (PPV), along with the breath amplitude accuracy metric weighted absolute percentage error (WAPE).

The primary signal quality concern for these sensors is the presence of false breaths during periods of disrupted breathing. Respectively, the sensitivity of BITalino, Shimmer, RespiBAN, and FLOW is $99.61 \%, 98.53 \%, 98.41 \%$, and $98.91 \%$. Their PPV is $96.28 \%, 96.58 \%$, $90.81 \%$, and $98.81 \%$. Finally, their WAPE is $13.82 \%, 16.89 \%, 13.60 \%$, and $8.75 \%$. The supine position is showing the overall best signal quality for all sensors, and the abdominal signal shows a better signal quality than the thoracic signal for BITalino.

There are at least two important research questions to be investigated in future work. For example, the relationship between the metrics we employ and the performance of data mining classifiers for sleep apnea detection must be studied further. We employ no explicit noise filtering in this work, so another example is to study further if and how much the signal quality can be improved by various noise filtering techniques.

\section{ACKNOWLEDGMENTS}

This work has been supported by the Cesar project (The Norwegian Research Council of Norway, project number 250239). 


\section{REFERENCES}

[1] Richard B. Berry and Mary H. Wagner. 2014. Sleep Medicine Pearls, 1-690. IsBN: 9781455770519.

[2] Richard B. Berry et al. 2012. Rules for scoring respiratory events in sleep: Update of the 2007 AASM manual for the scoring of sleep and associated events. Journal of Clinical Sleep Medicine, 8, 5, 597-619. IsSN: 15509389. DOI: $10.5664 /$ jcsm. 2172 .

[3] biosignalsplux. 2018. Respiban researcher. Retrieved Mar. 1, 2018 from http: //biosignalsplux.com/en/respiban-researcher.

[4] BITalino. 2018. Plugged kit ble. Retrieved Mar. 1, 2018 from http://bitalino.com/ en/plugged-kit-ble.

[5] J. P. Cantineau, P. Escourrou, R. Sartene, C. Gaultier, and M. Goldman. 1992. Accuracy of respiratory inductive plethysmography during wakefulness and sleep in patients with obstructive sleep apnea. Chest, 102, 4, 1145-1151. ISSN 00123692. Dor: 10.1378/chest.102.4.1145.

[6] Harald Hrubos-Strøm et al. 2011. A Norwegian population-based study on the risk and prevalence of obstructive sleep apnea The Akershus Sleep Apnea Project (ASAP). fournal of Sleep Research, 20, 1 PART II, 162-170. ISSN: 09621105. DOI: $10.1111 / \mathrm{j} .1365-2869.2010 .00861 . x$.

[7] K Konno and Jere Mead. 1967. Measurement of the separate volume changes of rib cage and abdomen during breathing. Fournal of applied physiology (Bethesda, Md. : 1985), 22, 3, 407-422. ISSN: 0021-8987.

[8] Stein Kristiansen, Mari Sønsteby Hugaas, Vera Goebel, Thomas Plagemann, Konstantinos Nikolaidis, and Knut Liestøl. 2018. Data Mining for Patient Friendly Apnea Detection. submitted to IEEE Access, May 2018

[9] NOX Medical. 2018. Nox t3. Retrieved Jan. 27, 2018 from http : // www noxmedical.com/products/nox-t3-sleep-monitor

[10] N. M. Punjabi. 2008. The Epidemiology of Adult Obstructive Sleep Apnea. Proceedings of the American Thoracic Society, 5, 2, (Feb. 2008), 136-143. ISSN: 1546-3222. DoI: 10.1513/pats.200709-155MG.

[11] Shimmer. 2018. Shimmer ecg. Retrieved Jan. 27, 2018 from http://www . shimmersensing.com/products/ecg-development-kit.

[12] SweetZpot. 2018. Flow. Retrieved Apr. 25, 2018 from https://www.sweetzpot. com/flow.

[13] Manjari Tripathi. 2008. Technical notes for digital polysomnography recording in sleep medicine practice. Annals of Indian Academy of Neurology, 11, 2, (Apr. 2008), 129-138. ISSN: 1998-3549. DOI: 10.4103/0972-2327.41887.

[14] K F Whyte, M Gugger, G A Gould, J Molloy, P K Wraith, and N J Douglas. 1991. Accuracy of respiratory inductive plethysmograph in measuring tidal volume during sleep. Journal of applied physiology (Bethesda, Md. : 1985), 71, 5, (Nov. 1991), 1866-1871. ISSN: 01617567. 